$\begin{array}{ll}\text { Research Square } & \text { Preprints are preliminary reports that have not undergone peer review. } \\ \text { They should not be considered conclusive, used to inform clinical practice, } \\ \text { or referenced by the media as validated information. }\end{array}$

\title{
Symptomatic treatment (using NSAIDS) versus antibiotics in uncomplicated lower urinary tract infection: a meta-analysis and systematic review of randomized controlled trials
}

Albert Macaire Chua Ong Lopez ( $\square$ macaire014@gmail.com )

St. Luke's Medical Center-Quezon City

Charles Jeffrey L. Tan

St. Luke's Medical Center-Quezon City

Antonio S. Yabon II

St. Luke's Medical Center-Quezon City

Armin N. Masbang

St. Luke's Medical Center-Quezon City

Research article

Keywords: Symptomatic treatment, NSAIDS, antibiotics, uncomplicated UTI

Posted Date: January 14th, 2020

DOI: https://doi.org/10.21203/rs.2.20800/v1

License: (1) This work is licensed under a Creative Commons Attribution 4.0 International License. Read Full License 


\section{Abstract}

Background: Current guidelines recommend empiric antibiotics as first-line treatment for uncomplicated UTI. Despite proven benefits in treatment, antibiotic resistance rates still rise.

Objectives: This meta-analysis aims to determine whether NSAIDs can serve as an effective and safe option in the treatment of uncomplicated lower UTI among non-pregnant women compared to antibiotics.

Data Sources: PUBMED,MEDLINE,EMBASE,CENTRAL,ACP databases and other grey literature sources from inception to June 2019 were sought in the relevant search.

Study Eligibility Criteria, Participants and Intervention: Randomized controlled trials that compare the use of NSAIDs versus antibiotics in non-pregnant women $\geq 18$ years old with uncomplicated urinary tract infection.

Methods: Primary outcomes were symptom resolution of UTI at Day 3 or 4 of intervention,and upper UTI complications. Secondary outcomes include persistence of positive urine culture despite treatment and need for another rescue antibiotic. Random and fixed-effects model for dichotomous data using Mantel-Haenszel and Peto odds method were reported at $95 \% \mathrm{Cl}$ followed by sensitivity analysis with substantial heterogeneity.

Results: The probability of having a symptom resolution by Day 3 or 4 with NSAID use is only less than three-fourths of that with antibiotic treatment(RR: $0.69 ; 95 \% \mathrm{Cls}[0.55,0.86]$, I $2=73$ ). The odds of developing upper UTI complications with use of NSAIDs are 6.49 to 1 for antibiotics (Peto OR: 6.49, 95\% Cls [3.02, 13.92], I $2=0 \%$ ).Secondary analysis showed that the NSAID group is $2.77 x$ more likely to have persistence of a positive microbiologic urine culture than the antibiotic group (RR: $2.77 ; 95 \%$ Cls [1.95, 3.94], I $2=36 \%$ ). Treatment with NSAIDs are three times more likely to use a secondary or rescue antibiotic due to persistent or worsening symptoms as compared to antibiotics (RR: $3.10 ; 95 \%$ Cis [2.17, 4.43], I 2 =53\%).

Conclusion: This meta-analysis could not prove non-inferiority of NSAIDs over antibiotics. However,the authors would still recommend maintaining the use of empiric antibiotic therapy as the primary treatment for UTI because of limited NSAID benefits with complication risks.

\section{Background}

Urinary tract infection remains as one of the leading causes of morbidity worldwide, with an estimated prevalence of $0.7 \%$ [1]. An estimated $50-60 \%$ of women report having UTI in their lifetime [2]. In the United States alone, UTIs account for nearly 7 million office visits, 1 million emergency department visits, and more than 100,000 hospitalizations with an annual healthcare expenditure cost of at least 1.6 billion dollars [3].

Clinically, UTIs can be categorized as either uncomplicated or complicated. Uncomplicated UTI is further differentiated into lower UTI or cystitis, and upper UTI or pyelonephritis. Uncomplicated lower UTI is suspected in mostly premenopausal, non-pregnant women presenting with acute symptoms of dysuria, urgency, frequency, lower abdominal pain and absence of fever without significant structural or functional abnormalities within the urinary tract.

Current primary care guidelines recommend empiric antibiotics as the first line of treatment for uncomplicated urinary tract infection [4]. A meta-analysis of randomized controlled trials showed superiority of antibiotic therapy over placebo in treatment of adult non-pregnant women with uncomplicated cystitis $\left(\mathrm{OR}=4.67,95 \% \mathrm{Cls}[2.34,9.35], \mathrm{I}^{2}=58 \%\right)$ [5].

Despite the benefits of antibiotic treatment in achieving symptomatic and bacteriologic cure, antibiotic resistance rates are on the rise. The most common pathogen is uropathogenic E. coli (UPEC). In a recent study done in Southern China primary care, antibiotic prescription rate is at $82.2 \%$. Isolates of E.coli revealed resistance rates to ampicillin, co-trimoxazole, ciprofloxacin, amoxicillin and nitrofurantoin at $59.8 \%$, $31.8 \%, 23.4 \%, 1.9 \%$ and $0.9 \%$ respectively [6]. In addition, antibiotic adverse event resulting in health service use and cost can't be undermined. Oral sulfonamides (23.2\%; $20.6-25.8 \%)$, penicillin $(20.8 \% ; 19.3-22.4 \%)$, and quinolones $(15.7 \% ; 14.2-17.1 \%)$ were the 3 most common antibiotic classes that are implicated in emergency visits due to antibiotic associated adverse effects. Rashes and pruritus were the commonly documented adverse reactions across all antibiotics and majority is due to sulfonamides. [7]

With the advent of antimicrobial stewardship, combating the overuse of antibiotics and dealing with increasing resistance rates, it is essential to determine if there are other efficacious alternative options to antibiotics in the treatment of uncomplicated lower urinary tract infections. 
One of the strategies is to delay institution of antibiotics. In the study by Little et. al., there were no significant differences in the severity of symptoms for 2 to 4 days after seeing a health professional between immediate vs delayed antibiotics (at least 48 hrs) (mean frequency of symptoms on a 0 to 6 scale: immediate antibiotics 2.15 , delayed antibiotics $2.11 ; p=0.177$ ). However, patients who delayed starting antibiotics for at least $48 \mathrm{hrs}$ had symptoms of $37 \%$ longer duration than those taking immediate antibiotics (incidence rate ratio $1.37,95 \%$ Cls [1.11,1.68], $p=0.003)[8]$.

Another alternative strategy is symptomatic treatment with non-steroidal anti-inflammatory drug. This is based on studies which suggest uncomplicated urinary tract infection is a self-limiting condition, with a favorable natural course of the disease. [9-11] Several recent randomized controlled trials have compared symptomatic treatment using non-steroidal anti-inflammatory drugs to antibiotics in the treatment of uncomplicated UTI. A 2010 landmark pilot randomized controlled trial by Bleidorn et.al. with 79 participants revealed that on Day 4 of illness, the ibuprofen group reported fewer symptoms than the ciprofloxacin group (symptom resolution $58.3 \%$ vs $51.5 \%$; $p=0.744$ ), which then supported the assumption of non-inferiority of NSAIDS compared to antibiotics in the treatment of symptomatic uncomplicated UTI [12]. Three other randomized controlled studies followed, with conflicting results [13,14,15].

In this paper, we performed a meta-analysis to assess whether the use of NSAIDs can serve as an effective and safe alternative option in the treatment of uncomplicated lower urinary tract infection among non-pregnant women as compared to antibiotics.

\section{Research Question}

Among non-pregnant women with symptoms of uncomplicated lower urinary tract infection, how effective and safe is treatment with nonsteroidal anti-inflammatory drugs (NSAIDs) compared to antibiotics in achieving symptomatic cure using a meta-analysis of randomized controlled trials?

\section{Specific Objectives}

The general objective of this meta-analysis is to determine the efficacy of non-steroidal anti-inflammatory drugs as compared to antibiotics in achieving symptomatic cure for uncomplicated lower urinary tract infection in non-pregnant women. Our specific objectives include assessment of complications of NSAIDs compared to antibiotics on a) upper UTI infection which comprised of febrile UTI and pyelonephritis, b) follow-up urine microbiologic cultures, and c) use of secondary antibiotics due to persistent or worsening symptoms of UTI.

\section{Methods}

\section{Criteria for considering studies for this review}

Studies were selected on the basis of a randomized-controlled study design, regardless of blinding status, or a specific study design such as cluster-randomized or cross-over type. Pre-specified inclusion criteria for type of participants comprised of non-pregnant women aged > 18 yrs old with one or more signs or symptoms of acute uncomplicated lower urinary tract infection: urinary frequency, dysuria, urgency, hematuria or suprapubic pain. Only included in this review are those RCTs that compare use of interventional non-steroidal anti-inflammatory drugs or NSAIDs versus antibiotics as active control. The type of NSAID or antibiotic drug, dosage/intensity, frequency and duration were not restricted in our inclusion criteria.

Primary outcomes for inclusion were 1) symptom resolution by Day 3 or 4 of treatment as defined by the respective studies, and 2) complications of primary treatment defined as progression to upper urinary tract infection subdivided as a) acute pyelonephritis presenting as fever with flank pain or costovertebral tenderness and urinary symptoms of dysuria, frequency, urgency, hematuria or suprapubic pain, b) febrile UTI manifesting as fever with urinary symptoms of frequency, dysuria, urgency, hematuria or suprapubic pain but without back pain, flank pain or CVA tenderness.

Secondary outcomes were patients with 1) persistence of a positive urine culture defined as growth of bacterial isolate on culture media after a maximum of 4 days of primary treatment and $b$ ) secondary treatment with new antibiotics defined as use of a rescue antibiotic after primary treatment with non-steroidal anti-inflammatory drugs or the need to switch to another antibiotic class group from an initial different antibiotic therapy due to persistent or worsening symptoms of UTI.

This study excluded non-randomized trials and participants that encompass pregnant women, the immunocompromised, recurrent urinary tract infection, and with an initial diagnosis of pyelonephritis or an upper urinary tract infection.

\section{Data sources and search methods for identification of studies}


This meta-analysis was performed in accordance to the evidence-based PRISMA (Preferred Reporting Items for Systematic Reviews and Meta-analyses) guidelines [16]. Three independent reviewers (A.M.C.-O.L., C.L.-T., and A.S.-Y.) performed a relevant search and evaluation of English-language randomized controlled trials on non-steroidal anti-inflammatory treatment for uncomplicated urinary tract infection published from inception up to June 2019, in the following scientific search engines: PUBMED, Cochrane Central Register of Controlled Trials, ACP Journal Club. The search strategy used was "(non-steroidal anti-inflammatory drugs OR ibuprofen or diclofenac) AND (cystitis OR urinary tract infection OR uncomplicated urinary tract infection)". Unpublished trials and ongoing studies in national and international trial registers (clinical trials.gov, ISRCTN Register and WHO ICTRP), dissertation and thesis databases, conference abstracts and other grey literature sources (EAGLE, HMIC, NTIS) were also sought in the relevant search. Lists of potentially relevant randomized controlled trials were then screened and appraised thoroughly for inclusion in the meta-analysis. Reviewers independently extracted relevant information. Any disagreements or differences were resolved by consensus by the co-authors and consultation with a fourth expert investigator (A.N.-M.).

\section{Data collection}

Review authors have planned in advance the relevant data to be collected in this meta-analysis and systematic review. A data collection form was created which included the citation details, study design, total study duration, type and number of participants, study location, diagnostic criteria for its inclusion, baseline patient characteristics, description of the intervention and control, relevant outcome of interest, and results.

\section{Selection of studies}

Three authors (A.M.C.-O.L., C.L.-T., and A.S.-Y.) independently performed a systematic process for selecting studies for inclusion in the review. After merging through the search results, no duplicate records of the same report were identified. Manuscript titles and abstracts were examined carefully and included only those that met the criteria for this review. Subsequently, the reviewers independently retrieved the full text of the potentially relevant reports. The full text reports were then scrutinized for compliance of studies with the eligibility criteria. If there are missing data results or inconsistencies, the reviewers attempted to correspond with the original investigators. Any clarification or discrepancies in the study selection process were resolved accordingly by the authors via a consensus.

\section{Data extraction and management}

Five data extractors, an infectious disease specialist, a fellow resident-in-training, and three of the review authors (A.M.C.-O.L., C.L.-T., and A.S.-Y.) independently extracted data from every report using a data collection form created by the authors. Any disagreements were resolved by reviewing again the data, and through consensus.

\section{Assessment of risk of bias in included studies}

For each trial, reviewers independently adopted the Cochrane Risk of Bias tool 5.2.0 to judge the adequacy of randomization, allocation concealment, blinding of participants, personnel, assessors, outcome-data completeness, and to check for selective outcome reporting and other possible sources of bias. Reviewers assessed the risk of bias in each of these domains as "high risk", "low risk", or "unclear." Any discrepancies in risk assessment were settled through constructive discussions between authors.

\section{Data synthesis and analysis}

Data synthesis and analysis was performed using Revman 5.3.5 for Windows 10. The effect measure of choice was stated as risk ratio for dichotomous data reported at 95\% confidence interval. However, in dichotomous outcomes with zero-event rates, the Peto one-step odds ratio was then reported. A 2-sided $p<0.05$ was determined as statistically significant.

Statistical heterogeneity between randomized controlled trials were assessed using the $\mathrm{I}^{2}$ statistics, with an interpretation of $\mathrm{I}^{2}$ value of 30 $60 \%$ representing moderate heterogeneity, 50-90\% indicating substantial heterogeneity, and $75-100 \%$ interpreted as considerable heterogeneity. If heterogeneity was identified, the random-effects analysis model was used; otherwise, the fixed-effect estimates were reported. The Mantel-Haenszel method was used primarily in the analysis in consideration for some studies with small sample sizes and lower event rates. On the other hand, the Peto's method was performed for dichotomous outcome data with zero cell counts. Assessment of publication or reporting bias was evaluated using funnel plot.

When significant heterogeneity was still detected even after exploring the use of random-effects model, a sensitivity analysis was performed by doing a systematic approach of repeating the initial analysis through exclusion of studies that are deemed not eligible on the basis of sample size, methodological quality, variances on patient population, intervention features and data . A L'Abbé plot was likewise used for investigating the potential sources of heterogeneity. Sources of heterogeneity were identified and discussed in this review. 


\section{Results}

\section{Search results and description}

Out of the 367 records that were identified through database searching. 359 studies were excluded due to lack of relevant information from the predefined criteria. After full review of the remaining 8 studies, 4 were excluded. Moore et al. [17] used uva-ursa extract with ibuprofen for symptomatic treatment of urinary tract infection but comparison was not made with antibiotics at the start of the study. Aloush et al [18] did not specify whether the urinary tract infection had lower or upper urinary tract infection. Patients included had symptoms of flank pain suggestive of upper urinary tract infection. Jamil et al [19] compared both potassium citrate and flurbiprofen with ciprofloxacin. Including potassium citrate will affect the heterogeneity in the analysis. Similarly, Ko et. al [20] compared both NSAID with antibiotics and antibiotics. Four randomized controlled trials were deemed eligible for quantitative synthesis (Figure 1). The trials were specifically designed to investigate the use of non-steroidal anti-inflammatory drugs in uncomplicated UTI. All trials reported outcomes of urinary tract symptoms after treatment with NSAID versus antibiotic as symptom burden and/or resolution. Three trials reported on resolution of UTI symptoms at day 4 with day of randomization defined as day $0[12,13,15]$, while only one trial reported symptom resolution on day 3 , however its day of randomization was defined as day 1 [14]. Three studies evaluated on the after-treatment complication rates of upper UTI described as febrile UTI and/or pyelonephritis. Three trials reported on the presence of positive urine cultures on follow-up with one study on Day 7, 10 and 14 respectively. All trials did report on the need for secondary antibiotic treatment for persistent UTI symptoms.

[Figure 1]

\section{Risk of bias in included studies}

Risk of bias assessment of individual trials were summarized in Figure 2. All trials employed computer-generated randomization sequence in varying block sizes of 6 in two studies, 4 and 6 in one study, and 2, 4, 6 or 8 in another study.

Allocation concealment of all trials was adequate prior to assignment. Two trials kept the randomization list in a sealed opaque envelope. One trial concealed the allocation with sealed, sequentially numbered opaque containers of identical appearance. The other trial kept the randomization list at an independent Norwegian pharmaceutical company and was retrieved only at the end of the study.

All studies performed a double-blinded study design. The study participants, treating doctor, study nurses and outcome assessors were blinded from knowledge of which intervention the participant received.

Overall, two trials were assessed as high risk for attrition bias and two trials were judged to be at low risk of bias. The pilot study by Bleidorn et. al. [12] used a per protocol analysis with exclusion of 9 participants due to incomplete symptom data and 3 participants with finding of exclusion criteria after inclusion (out of 80 patients randomized to trial treatment). The research study by Gágyor et. al. [15] partly addressed the incomplete outcome data with use of intention to treat analysis accounting for 19 out of the 484 women who were lost to follow-up and 9 patients who withdrew from the trial. Conversely, the Kronenberg trial [14] was reviewed to have a low risk for attrition bias. An intention to treat analysis was performed with missing values accounted for by multiple imputation. The Vik trial [15] was judged to be of high risk of attrition bias since 24 drop-out patients out of the total 383 randomized women were excluded in both intention to treat and per protocol analysis.

Reporting bias due to selective outcome reporting was noted in the pilot study by Bleidorn et.al.[12] as the breakdown of serious and nonserious adverse events was not fully specified such as UTI complications of pyelonephritis. Isolation of resistant strains post-treatment was also not reported among the studies.

[Figure 2-8]

\section{Outcomes of the meta-analysis}

Across 4 trials, a total of 1,144 participants were included, with 584 in the NSAID group and 560 in the antibiotic group.

Four studies in total were investigated on whether symptomatic treatment with non-steroidal anti-inflammatory drugs is non-inferior or equivalent to antibiotics in the treatment of uncomplicated lower urinary tract infection in women.

The probability of having a symptom resolution by Day 3 or 4 with NSAID use is only less than three-fourths of that with antibiotic treatment (RR: $0.69,95 \%$ Cls $[0.55,0.86], I^{2}=73$ ) (Figure $3 \mathrm{~A}$ ). Between-trial heterogeneity was identified in this analysis with an $\mathrm{I}^{2}$ of $73 \%$ and as reflected by the L'Abbé plot (Figure 11). The dashed line runs through the bottom-right sector of the L'Abbé plot signifying that the pooled effect estimate favors the antibiotics group in terms of symptom resolution by Day 3 or 4 . The pilot study by Bleidorn et. al. [12] lies above the line 
of equality, while most studies fall near the dashed line. This suggests that the Bleidorn et. al. study may have contributed to the betweenstudy heterogeneity, which distorted the overall pooled effect. A sensitivity analysis was subsequently performed, which showed a decrease in $\mathrm{I}^{2}$ from $73 \%$ to $56 \%$ (Figure 3B) after the exclusion of the pilot 2010 study by Bleidorn et. al [12], but still with moderate heterogeneity. The major source of heterogeneity identified in this analysis is the difference in their methodology on the definition of symptom resolution. The Bleidorn et. al. and Gágyor et. al. study group defined symptom resolution as the number of patients with a symptom sum score of 0 (dysuria, frequency/urgency, and lower abdominal pain). Each symptom was graded on a scale from 0 (none) to 4 (very strong). Kronenberg et. al. used a questionnaire with five severity UTI symptoms (dysuria, frequency, urgency, abdominal pain, pain in lower back) categorized on a scale from 0 to 6 . Symptom resolution was defined on the basis of 2 or less points. On the other hand, Vik et. al. defined symptom resolution as the proportion of patients who felt cured on Day 4 with a symptom sum score of 0 using a patient diary. Its sum score consisted of dysuria, urinary frequency, and urgency graded on a scale from 0 (normal) to 6 (as bad as it could be). Other sources of heterogeneity include use of non-validated questionnaire as stated by the respectives studies, and differences in baseline symptom sum scores which could underestimate or overestimate the results.

Another primary outcome in this study showed that the odds of developing upper UTI complications (comprising of pyelonephritis and febrile UTI) with the use of NSAIDs are 6.49 to 1 for antibiotics. (Peto OR: 6.49, 95\% Cls [3.02, 13.92], I²=0\%) (Figure 4). Secondary outcome analysis showed that the NSAID group is 2.77 times more likely to have persistence of a positive microbiologic urine culture than the antibiotic group even after the initial treatment (RR: 2.77, 95\% Cls [1.95, 3.94], I ${ }^{2}=36 \%$ ) (Figure 5). Moreover, treatment with NSAIDs are three times more likely of having a secondary or rescue antibiotic due to persistent or worsening symptoms as compared to antibiotics (RR: $3.10,95 \%$ Cls [2.17, $4.43], I^{2}=53 \%$ ) (Figure 6). No significant publication bias was detected as based from the symmetric finding in the funnel plot (Figure 7 ).

\section{Discussion}

\section{Summary of Individual Studies}

This systematic review evaluated evidence from four European double-blinded randomized control trials involving 1144 patients with uncomplicated urinary tract infection conducted between 2007 to 2016.

The 2010 pilot study by Bleidorn et. al. analyzed 79 otherwise healthy women presenting with uncomplicated UTI symptoms, who were randomly assigned to either ibuprofen or ciprofloxacin for 3 days. Results supported the assumption that ibuprofen is non-inferior as compared to ciprofloxacin for treatment of uncomplicated UTI regarding symptom resolution and symptom course. However, there were no significant differences between Ibuprofen and ciprofloxacin ( $58.3 \%$ vs $51.5 \%$; $p=0.744$ ) as for symptom resolution on Day 4 . Conversely, the proportion of patients requiring secondary antibiotic treatment due to persistent or worsening symptoms was not statistically significant between the Ibuprofen and the Ciprofloxacin group (33.3 \% vs $18.2 \%, p=0.247$ ) [2]. Even with these trial results, the pilot study was limited by its inadequately powered study design with small number of participants. This triggered a series of pivotal trials with a determination of non-inferiority of NSAIDs compared with antibiotics for symptom control and treatment.

The Immediate versus Conditional treatment of Urinary Tract Infection (ICUTI) double blinded randomized multicenter trial by Gágyor et. al. recruited 494 patients and randomly assigned them to treatment with a single dose of fosfomycin or ibuprofen for 3 days. The study assessed whether the antibiotic prescriptions for UTI can be reduced by symptomatic treatment with ibuprofen, without a significant increase in symptoms, recurrences or complications. Data showed that the ibuprofen group received significantly lower antibiotic courses compared to the fosfomycin group with an incidence rate reduction of $66.5 \%$ ( $95 \%$ Cls [58.8\%, 78.4\%]; $p<0.001)$. However, during the follow-up period, significantly more women in the Ibuprofen group (34\%) received antibiotic prescriptions compared to the fosfomycin group (14\%) (mean difference $21.1,95 \%$ Cls $[13.8,28,7], p<0.001)$ due to worsening or persistent symptoms. The findings are similar with the pilot study. Symptom relief of UTI was also less effective. A significantly higher total burden of symptoms and impairment of activity were observed in the Ibuprofen group and more cases of pyelonephritis. Though, nearly two-thirds of women treated symptomatically with Ibuprofen recovered without any antibiotic treatment [13]. A follow-up analysis of the ICUTI trial showed that there is no difference in rates of recurrent UTI between antibiotic and non-antibiotic treatment beyond four weeks after initial treatment [24].

The next trial by Kronenberg et. al. involved 253 women with uncomplicated lower UTI and they were randomly assigned to receive diclofenac or norfloxacin for 3 days. Results revealed symptom resolution at Day 3 were more common among women who took norfloxacin (80\%) versus those assigned to diclofenac (54\%) (risk difference $27 \%, 95 \%$ Cls $[15 \%, 38 \%], p<0.001$ ). A higher incidence of pyelonephritis (5\%) was also observed in the diclofenac group (risk difference $5 \%, 95 \%$ Cls $[1 \%, 8 \%], p=0.031$ ). On the beneficial side, similar with the study results by Gagyor et. al., antibiotic use can be lessened as women who received diclofenac were $37 \%$ less likely to use any antibiotic until day 30 after the randomization (risk difference $37 \%, 95 \%$ Cls [28\%, 46\%], $p<0.001$ [14]. 
The 2017 trial conducted by Vik et. al. enrolled 383 women who were randomly allocated to treatment with either ibuprofen or pivmecillinam for 3 days. It concluded that ibuprofen was inferior to pivmecillinam for treating uncomplicated UTIs. Despite that more than half of women treated with ibuprofen recovered without antibiotics, this came at a cost of higher symptom burden with only $38.7 \%$ of the lbuprofen group felt cured by Day 4 versus $73.6 \%$ in pivmecillinam group (risk difference $35 \%, 90 \%$ Cls [27\%, 43\%]; $p>0.99$ ), followed by longer duration of symptoms (6 days vs 3 days), and higher rate of pyelonephritis at 3.9\%[15].

\section{Summary of Evidence}

Consolidating all the findings above, this meta-analysis determined that the treatment of uncomplicated UTI with non-steroidal antiinflammatory drugs does not substantially improve symptom burden and achieve symptomatic cure, compounded further by complication risks as compared to standard antibiotic use. Our sensitivity analyses showed that the effectiveness of symptom resolution is less with NSAID treatment than use of antibiotics (RR: $0.63,95 \% \mathrm{Cls}[0.53,0.74], I^{2}=56$ ). Moreover, the pooled number needed to harm is 4.76 for persistence of symptoms on Day 3 or 4 of illness ( $\mathrm{NNH} 4.76,95 \% \mathrm{Cls}[12.5,2.94]$ ), with a risk difference of 0.21 (RD $0.21,95 \% \mathrm{Cls}[0.08$, $\left.0.34], I^{2}=78\right)$ (Figure 3B).

The initial hypothesis of the possible use of NSAIDs in symptomatic treatment of uncomplicated urinary tract infections stems from the postulate that inflammation plays a key role in the development of lower urinary tract symptoms, with high sensitivity C-reactive protein as a marker of inflammation. Previous studies reported a significant association between CRP levels and lower urinary tract symptoms in women and men [22,23], and subsequently among urinary tract infection patients [24]. Theoretically, NSAIDs may reduce contraction of the bladder muscle, which is partly responsible for the lower urinary tract symptoms, by inhibiting expression of cyclooxygenase 2 , and synthesis of prostaglandins. This pathogenic mechanism was replicated in the study by Takagi-Matsumoto et. al., which revealed dose-dependent suppression of rhythmic contraction induced by distension on normal and cystitis rats with intravenous administration of aspirin, indomethacin or ketoprofen [25]. However, it did not translate well in the clinical setting.In the 2019 ATAFUTI trial, there was no evidence of differences between those who took Ibuprofen vs. placebo in terms of symptom severity after 2-4 days of intervention (LS mean -0.01, 95\% $\mathrm{Cl}[-0.27,0.26], \mathrm{p}=0.951)$ [17]. Also, in a randomized controlled pilot study by Ko et. al., no significant difference was observed in the degree of pain scale reduction between those who took cepodoxime plus aceclofenac versus cepodoxime alone on Day 3 of treatment $(p=0.134)$ [20]

The non-steroidal anti-inflammatory drugs are known for its anti-inflammatory, analgesic and anti-pyretic properties. Nevertheless, some studies have demonstrated moderate to strong in vitro antimicrobial activity when tested against different bacterial isolates of Gram-positive and Gram-negative organisms. Aspirin inhibited all of the $S$. aureus and $E$. faecalis isolates of UTI at the most effective concentration of 500 $\mathrm{ug} / \mathrm{ml}$ [26]. Conversely, diclofenac exhibited in vitro inhibition on $67 \%$ of clinically isolated strains of E.coli, with MIC values ranging from 5$50 \mathrm{ug} / \mathrm{ml}$ [27]. In case of Ibuprofen, zones of inhibition were observed for S. aureus, B.subtilis, C. albicans and A. brasiliensis, hence signifying a broad spectrum of activity for bacterial and fungal strains [28]. However, contrary to the previous studies, a recent investigation revealed that Ibuprofen lacks direct antimicrobial properties for treatment of urinary tract infection isolates without any effect on bacterial growth of E. coli or E. faecalis [29]. In our study, results revealed that the non-steroidal anti-inflammatory group is more likely to have a persistence of a positive microbiologic urine culture compared to use of antibiotics after treatment (RR: $2.77,95 \%$ Cls [1.95, 3.94], I²=36\%). Failure to reach a bacteriologic cure can delay or not effectively attain optimal symptom improvement. This was shown in one of the reviewed studies by Vik et. al. as patients in the ibuprofen group who have a positive culture had a higher symptom burden and longer duration of lower urinary tract symptoms than those with a negative culture [15].

Another key finding in this study showed that while $43.34 \%$ of women in the combined trials treated with NSAIDs achieved symptomatic cure, the observed odds of developing upper UTI complications (pyelonephritis, and febrile UTI) was significantly higher in the NSAID group (Peto OR: $6.49,95 \% \mathrm{Cls}[3.02,13.92], \mathrm{I}^{2}=0 \%$ ). This was due to the delay in instituting antibiotics. Due to the persistence or worsening of symptoms, patients who were given NSAID initially needed secondary treatment with antibiotics (RR: $3.10,95 \%$ Cls $[2.17,4.43], I^{2}=53 \%$ ). In the study by Vik et. al., some even required hospitalization for IV antibiotics (2 out of 7 with serious adverse events) [15]. While it is an important outcome that at least $40 \%$ of patients were spared of antibiotics, we cannot disregard those who were harmed during the process.

These results suggest that NSAIDS are not comparable to antibiotic treatment for uncomplicated UTI. Giving NSAIDs can reduce the use of antibiotics [13], but at the expense of high symptom burden, longer duration of symptoms, and more cases of progression to pyelonephritis.

Previous studies have compared the use of placebo and antibiotics in uncomplicated UTI favoring the administration of antibiotics due to the worsening of complications in the other placebo arm [5]. The same conclusion can be made in this study.

Other antibiotic sparing strategies for uncomplicated UTI 
Herbal preparations has also been used as part of alternative therapy sparing antibiotic use. In the 2019 ATAFUTI study [17], it investigated on the use of uva-ursi leaf extract in relieving urinary symptoms and reduction in consumption of antibiotics. There was no difference in symptom severity between uva-ursi and placebo (LS mean $-0.06,95 \%$ Cls $[-0.33,0.21], p=0.661$ ), and likewise, no statistical significance in terms of antibiotic reduction (OR 0.59, 95\% Cls [0.22,1.58], $p=0.293$ ). Cranberries has also been used traditionally in treating UTI as well as from its recurrence among healthy women. However, a metaanalysis showed that cranberry products did not significantly reduce the occurrence of symptomatic UTI overall (RR 0.86, 95\% Cl [0.71, 1.04], I² $=55 \%$ ) [30].

\section{Strengths and Limitations}

This manuscript is the first meta-analysis with systematic review of NSAID vs antibiotics for treatment of uncomplicated UTI. However, this study is limited only to search titles or abstracts of English language articles. Other potential sources of bias in each study remain to be elucidated which may affect the magnitude of effect estimate. There is also a paucity of randomized controlled trials available for this metaanalysis.

\section{Conclusion}

This meta-analysis could not prove non-inferiority of NSAIDs over antibiotics. However, the authors would still recommend maintaining the use of empiric antibiotic therapy as the primary treatment for urinary tract infection because of limited NSAID benefits in symptom resolution, compounded by complication risks of developing upper UTI, with presence of positive urine culture even after treatment, and the need to use a secondary or rescue antibiotic. Despite the limited benefits of NSAIDs, there are at least a subgroup of patients who are responsive with spontaneous resolution of symptoms without a need for antibiotics. Future studies should emphasize on identifying aspects of underlying predictors and moderators of treatment effects.

\section{Abbreviations}

NSAIDs

non-steroidal anti-inflammatory drugs

UTI

urinary tract infections

\section{Declarations}

Ethics approval and consent to participate: This study was ethically approved by the St. Luke's Medical Center-Quezon City Institutional Ethics Review Committee last February 28, 2019.

Consent for publication: Not applicable

Availability of data and materials: All data generated and/or analyzed in this study are included in this main article and its supplementary files.

Competing interests: The authors declare that they have no competing interests.

Funding: None of the authors received any external funding, grants, salaries, benefits or services from a third-party for any piece of submitted work.

Author's Contributions: All authors made substantial contributions to the manuscript. AOL conceived and designed the whole study. AOL, CJF, AY, AM went through the rigorous data collection process: performed search strategies, screened search results, organized and screened retrieved papers, appraised quality of papers, and extracted data. AOL and CJF were responsible for data synthesis, analysis and management, respectively. AOL, CJF, and AY co-wrote the first draft, second draft and the final manuscript, while AM supervised the whole review by ensuring the accuracy and completeness of the study. All authors read and approved the final paper to be submitted.

Acknowledgments: We would like to thank our beloved institution, St. Luke's Medical Center-Quezon City for the support and the institutional review board for accepting our study in accordance with the ethical principles. The preliminary results of the study was presented in a paper poster session in the 29th European Congress of Clinical Microbiology and Infectious Diseases held in Amsterdam, Netherlands on 13 - 16 April 2019.

\section{References}


1. Tandogdu Z, Wagenlehner F. Global epidemiology of urinary tract infections. Current Opinion in Infectious Diseases. 2016;29(1):73-79.

2. Al-Badr A, Al-Shaikh G. Recurrent Urinary Tract Infections Management in Women : A Review. Sultan Qaboos University Medical Journal. 2013;13(3):359-367.

3. Simmering J, Tang F, Cavanaugh J, Polgreen L, Polgreen P. The Increase in Hospitalizations for Urinary Tract Infections and the Associated Costs in the United States, 1998-2011. Open Forum Infectious Diseases. 2017;4(1).

4. Philippine clinical practice guidelines on the diagnosis and management of urinary tract infections in adults 2013 update. Task Force on Urinary Tract Infections, P. P. G. G. I. D.; 2013.

5. Falagas M, Kotsantis I, Vouloumanou E, Rafailidis P. Antibiotics versus placebo in the treatment of women with uncomplicated cystitis: A meta-analysis of randomized controlled trials. Journal of Infection. 2009;58(2):91-102.

6. Wong C, Kung K, Au-Doung P, Ip M, Lee N, Fung A et al. Correction: Antibiotic resistance rates and physician antibiotic prescription patterns of uncomplicated urinary tract infections in southern Chinese primary care. PLOS ONE. 2018;13(2):e0192466.

7. Geller A, Lovegrove M, Shehab N, Hicks L, Sapiano M, Budnitz D. National Estimates of Emergency Department Visits for Antibiotic Adverse Events Among Adults-United States, 2011-2015. Journal of General Internal Medicine. 2018;33(7):1060-1068.

8. Tate I. Effectiveness of Five Different Approaches in Management of Urinary Tract Infection: Randomised Controlled Trial. The Journal of Emergency Medicine. 2010;39(2):270.

9. Ferry S, Holm S, Stenlund H, Lundholm R, Monsen T. Clinical and bacteriological outcome of different doses and duration of pivmecillinam compared with placebo therapy of uncomplicated lower urinary tract infection in women: The LUTIW project. Scandinavian Journal of Primary Health Care. 2007;25(1):49-57.

10. Christiaens T, De Meyere M, Verschraegen G, Peersman W, Heytens S, De Maeseneer J. Randomised controlled trial of nitrofurantoin versus placebo in the treatment of uncomplicated urinary tract infection in adult women. The British journal of general practice : the journal of the Royal College of General Practitioners. 2002;52(482):729-734.

11. Kahan N, Chinitz D, Kahan E. Physician adherence to recommendations for duration of empiric antibiotic treatment for uncomplicated urinary tract infection in women: a national drug utilization analysis. Pharmacoepidemiology and Drug Safety. 2004;13(4):239-242.

12. Bleidorn J, Gágyor I, Kochen M, Wegscheider K, Hummers-Pradier E. Symptomatic treatment (ibuprofen) or antibiotics (ciprofloxacin) for uncomplicated urinary tract infection? - Results of a randomized controlled pilot trial. BMC Medicine. 2010;8(1).

13. Gágyor I, Bleidorn J, Kochen M, Schmiemann G, Wegscheider K, Hummers-Pradier E. Ibuprofen versus fosfomycin for uncomplicated urinary tract infection in women: randomised controlled trial. BMJ. 2015;:h6544.

14. Kronenberg A, Bütikofer L, Odutayo A, Mühlemann K, da Costa B, Battaglia M et al. Symptomatic treatment of uncomplicated lower urinary tract infections in the ambulatory setting: randomised, double blind trial. BMJ. 2017;:j4784.

15. Vik I, Bollestad M, Grude N, Bærheim A, Damsgaard E, Neumark T et al. Ibuprofen versus pivmecillinam for uncomplicated urinary tract infection in women-A double-blind, randomized non-inferiority trial. PLOS Medicine. 2018;15(5):e1002569.

16. Moher D, Liberati A, Tetzlaff J, Altman D. Preferred Reporting Items for Systematic Reviews and Meta-Analyses: The PRISMA Statement. PLoS Med; 2009.

17. Moore M, Trill J, Simpson C, Webley F, Radford M, Stanton L et al. Uva-ursi extract and ibuprofen as alternative treatments for uncomplicated urinary tract infection in women (ATAFUTI): a factorial randomized trial. Clinical Microbiology and Infection. 2019;25(8):973-980.

18. Aloush S, Al-Awamreh K, Abu Sumaqa Y, Halabi M, Al Bashtawy M, Salama F. Effectiveness of antibiotics versus ibuprofen in relieving symptoms of nosocomial urinary tract infection. Journal of the American Association of Nurse Practitioners. 2019;31(1):60-64.

19. Jamil M, Farooq U, Sultan B, Khan R. Role of symptomatic treatment in comparison to antibiotics in uncomplicated urinary tract infections. Journal of Ayub Medical College Abbottabad. 2016; 28(4), 734-37.

20. Ko K, Lee W, Oh C et al. A combination of antibiotics and non-steroidal anti-inflammatory drugs more beneficial than antibiotic monotherapy for the treatment of female acute uncomplicated cystitis? A randomized controlled pilot study. Urology Journal. 2018; 15(6), 365-369.

21. Bleidorn J, Hummers-Pradier E, Schmiemann G, Wiese B, Gágyor I. (2016). Recurrent urinary tract infections and complications after symptomatic versus antibiotic treatment: follow-up of a randomised controlled trial. German medical science : GMS e-journal. 2016 ; 14.

22. Hsiao S, Lin H, Kuo H. The role of serum C-reactive protein in women with lower urinary tract symptoms. International Urogynecology Journal. 2012; 23(7), 935-940.

23. Kupelian V, McVary K, Barry M, Link C, Rosen R, Aiyer L et al. Association of C-reactive protein and lower urinary tract symptoms in men and women: results from Boston Area Community Health survey. Urology. 2009; 73(5), 950-957. 
24. Yildiz B, Poyraz N, Cetin N, Kural N, Colak O. High sensitive C-reactive protein: a new marker for urinary tract infection, VUR and renal scar. European review for medical and pharmacological sciences. European Review for Medical and Pharmacological Sciences. 2013;17(19), 2598-2564.

25. Takagi-Matsumoto H, Ng B, Tsukimi Y, Tajimi M. Effects of NSAIDs on Bladder Function in Normal and Cystitis Rats: a Comparison Study of Aspirin, Indomethacin, and Ketoprofen. Journal of Pharmacological Sciences. 2004;95(4):458-465.

26. Akhter T, Baqai R, Aziz M. Antibacterial effect of NSAIDS on clinical isolates of urinary tract infection and diabetic foot infection. Pakistan journal of pharmaceutical sciences.2010; 23 (1), 108-113.

27. Mazumdar K, Dutta N, Dastidar S, Motohashi N,Shirataki Y.Diclofenac in the Management of E. coli Urinary Tract Infections. In Vivo. 2006; 20(5), 613-619.

28. Obad J, Šušković J, Kos B. Antimicrobial activity of ibuprofen: New perspectives on an "Old” non-antibiotic drug. European Journal of Pharmaceutical Sciences. 2015;71:93-98.

29. Whiteside S, Dave S, Reid G, Burton J. Ibuprofen lacks direct antimicrobial properties for the treatment of urinary tract infection isolates. Journal of Medical Microbiology. 2019;68(8):1244-1252.

30. Jepson R, Williams G, Craig J. Cranberries for preventing urinary tract infections. Cochrane Database of Systematic Reviews. 2012;.

\section{Table}

Table 1. Study characteristics of NSAID versus Antibiotic use in Uncomplicated UTI 


\begin{tabular}{|c|c|c|c|c|c|c|}
\hline $\begin{array}{l}\text { First } \\
\text { author, year }\end{array}$ & $\begin{array}{l}\text { Study design, } \\
\text { location }\end{array}$ & \begin{tabular}{|l} 
Study \\
population
\end{tabular} & Inclusion criteria & Exclusion criteria & $\begin{array}{l}\text { NSAID } \\
\text { Antibiotic }\end{array}$ & Outcomes \\
\hline $\begin{array}{l}\text { Bleidorn, } \\
2010\end{array}$ & \begin{tabular}{|l|} 
Multicenter, \\
double- \\
blinded, \\
pilot trial, \\
Germany
\end{tabular} & \begin{tabular}{|lr|}
\multicolumn{2}{|l|}{ Non-pregnant } \\
women & aged \\
$18-85$ & y/o, \\
with & \\
uncomplicated \\
UTI
\end{tabular} & \begin{tabular}{|l} 
At least one of the \\
main UTI \\
symptoms dysuria \\
and frequency
\end{tabular} & $\begin{array}{l}\text { Signs of upper UTI } \\
\text { symptoms (fever, } \\
\text { back pain) } \\
\text { Pregnancy } \\
\text { Comorbidities: DM, } \\
\text { CKD } \\
\text { GIT abnormalities } \\
\text { or past urinary } \\
\text { surgery, Urine } \\
\text { catheterization, } \\
\text { immunosuppressive } \\
\text { therapy } \\
\text { UTI within the last } \\
\text { two weeks } \\
\text { Current use of } \\
\text { antibiotics } \\
\text { NSAIDs; } \\
\text { History of GI ulcers } \\
\text { Epilepsy } \\
\text { Allergies }\end{array}$ & $\begin{array}{l}\text { Ibuprofen vs } \\
\text { Ciprofloxacin }\end{array}$ & $\begin{array}{l}\text { Primary: } \\
\text { Symptom } \\
\text { resolution on } \\
\text { Day 4 } \\
\\
\\
\text { Secondary: } \\
\text { Burden } \\
\text { symptoms of } \\
\text { Day 4 and, } \\
\text { symptom } \\
\text { resolution on } \\
\text { Day 7 and } \\
\text { frequency of } \\
\text { relapses until } \\
\text { Day 28, and } \\
\text { incidence of } \\
\text { adverse events }\end{array}$ \\
\hline $\begin{array}{l}\text { Gagyor, } \\
2015\end{array}$ & \begin{tabular}{|l|} 
Multicenter, \\
double- \\
blinded, RCT, \\
Germany
\end{tabular} & \begin{tabular}{|lr|}
\multicolumn{2}{|l|}{ Non-pregnant } \\
women & aged \\
$18-65$ & y/o, \\
with & \\
uncomplicated \\
UTI
\end{tabular} & 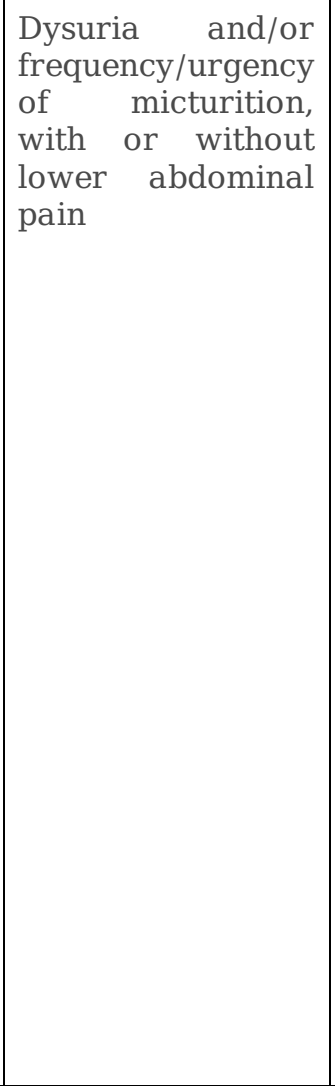 & $\begin{array}{l}\text { Any signs of upper } \\
\text { UTI (fever, loin } \\
\text { tenderness); } \\
\text { Pregnancy } \\
\text { Renal diseases } \\
\text { UTI within the past } \\
\text { two weeks } \\
\text { Urinary } \\
\text { catheterization. } \\
\text { Recent NSAID or } \\
\text { antibiotics use } \\
\text { History of GI ulcers } \\
\text { or severe acute or } \\
\text { exacerbated } \\
\text { chronic conditions }\end{array}$ & $\begin{array}{l}\text { Ibuprofen vs } \\
\text { Fosfomycin }\end{array}$ & 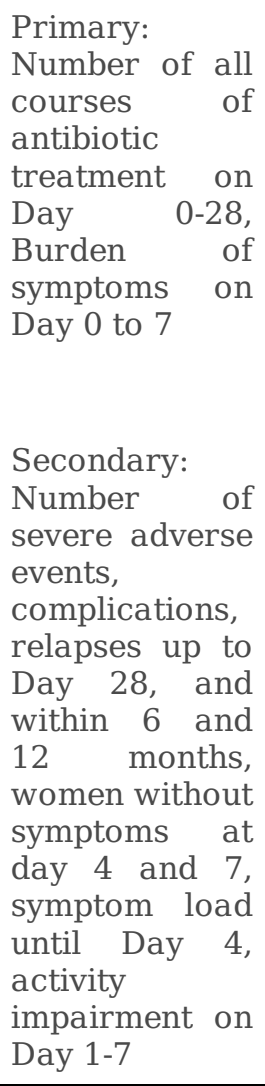 \\
\hline $\begin{array}{l}\text { Kronenberg, } \\
2017\end{array}$ & \begin{tabular}{|l|} 
Multicenter, \\
double- \\
blinded, RCT, \\
Switzerland
\end{tabular} & \begin{tabular}{|lr|}
\multicolumn{2}{|l|}{ Non-pregnant } \\
women & aged \\
$18-70$ & y/o, \\
with & \\
uncomplicated \\
UTI
\end{tabular} & \begin{tabular}{|lr} 
One or more \\
symptoms or signs \\
typical of acute \\
lower r \\
(dysuria, \\
frequency, \\
macrohematuria, \\
cloudy or smelly
\end{tabular} & $\begin{array}{l}\text { Pregnant women } \\
\text { and women } \\
\text { Signs of upper UTI: } \\
\text { (fever, } \\
\text { costovertebral pain } \\
\text { or tenderness, }\end{array}$ & $\begin{array}{l}\text { Diclofenac vs } \\
\text { Norfloxacin }\end{array}$ & $\begin{array}{ll}\text { Primary: } & \\
\text { Resolution } & \text { of } \\
\text { symptoms } & \text { at } \\
\text { day 3 } & \end{array}$ \\
\hline
\end{tabular}




\begin{tabular}{|c|c|c|c|c|c|c|}
\hline & & & $\begin{array}{l}\text { urine) or self- } \\
\text { diagnosed } \\
\text { symptomatic } \\
\text { cystitis (urine } \\
\text { dipstick was } \\
\text { positive for nitrite } \\
\text { or leucocytes, or } \\
\text { both) }\end{array}$ & 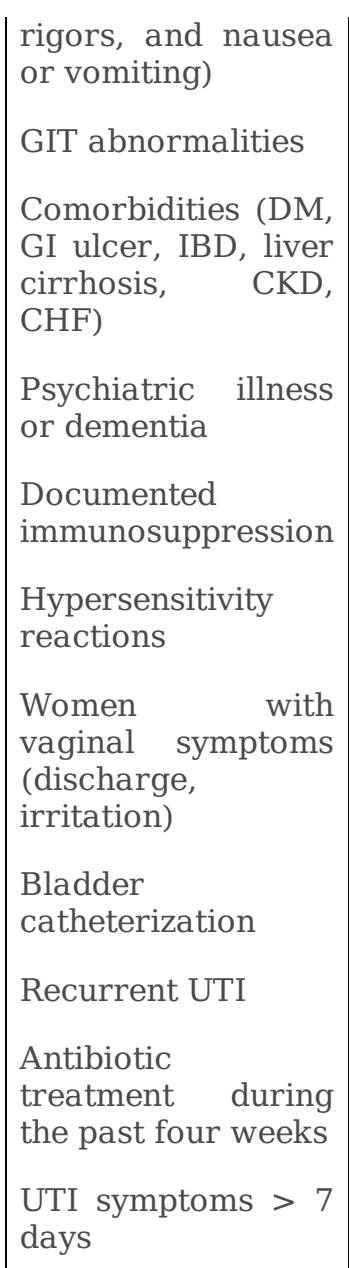 & & $\begin{array}{l}\text { Secondary: } \\
\text { Use of any } \\
\text { antibiotic up to } \\
\text { Day 30, } \\
\text { resolution of } \\
\text { symptoms on } \\
\text { Day } \\
\text { complete } \\
\text { absence of } \\
\text { symptoms on } \\
\text { Days } 3 \text { and } 7, \\
\text { use of rescue } \\
\text { antibiotic up to } \\
\text { Day } \quad 3, \\
\text { negative urine } \\
\text { culture result } \\
\text { on Day 10, } \\
\text { reconsultations } \\
\text { because of UTI } \\
\text { up to day 30, } \\
\text { adverse events, } \\
\text { serious } \\
\text { adverse events, } \\
\text { European } \\
\text { quality of life. }\end{array}$ \\
\hline Vik, 2018 & $\begin{array}{l}\text { Multicenter, } \\
\text { double- } \\
\text { blinded, } \\
\text { noninferiority, } \\
\text { RCT, Norway, } \\
\text { Denmark, } \\
\text { Sweden }\end{array}$ & $\begin{array}{l}\text { Non-pregnant } \\
\text { women aged } \\
18-60 \text { y/o, } \\
\text { with } \\
\text { uncomplicated } \\
\text { UTI }\end{array}$ & $\begin{array}{lr}\text { Dysuria } & \text { combined } \\
\text { with } & \text { either } \\
\text { increased } & \text { urinary } \\
\text { frequency } & \text { or } \\
\text { urinary } & \text { urgency } \\
\text { or both, } & \text { with or } \\
\text { without r } & \text { visible } \\
\text { hematuria } & \end{array}$ & 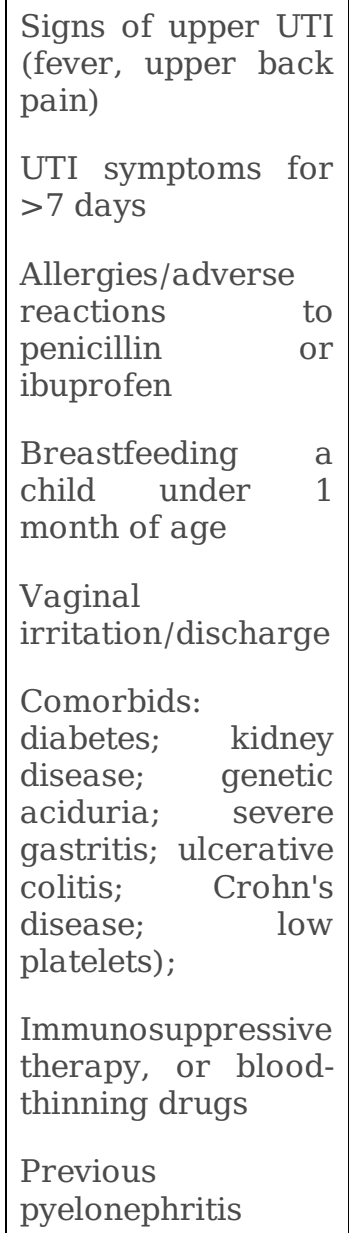 & $\begin{array}{l}\text { Ibuprofen vs } \\
\text { Pivmecillinam }\end{array}$ & $\begin{array}{l}\text { Primary: } \\
\text { Proportion of } \\
\text { patients who } \\
\text { felt cured by } \\
\text { Day } 4 \\
\\
\text { Secondary: } \\
\text { Proportion of } \\
\text { patients in } \\
\text { need of } \\
\text { secondary } \\
\text { treatment with } \\
\text { antibiotics, } \\
\text { proportion of } \\
\text { patient with } \\
\text { positive second } \\
\text { urine culture, } \\
\text { in need of a } \\
\text { medical } \\
\text { consultation } \\
\text { within } 4 \text { weeks } \\
\text { of follow-up, } \\
\text { cases } \quad \text { of } \\
\text { pyelonephritis }\end{array}$ \\
\hline
\end{tabular}




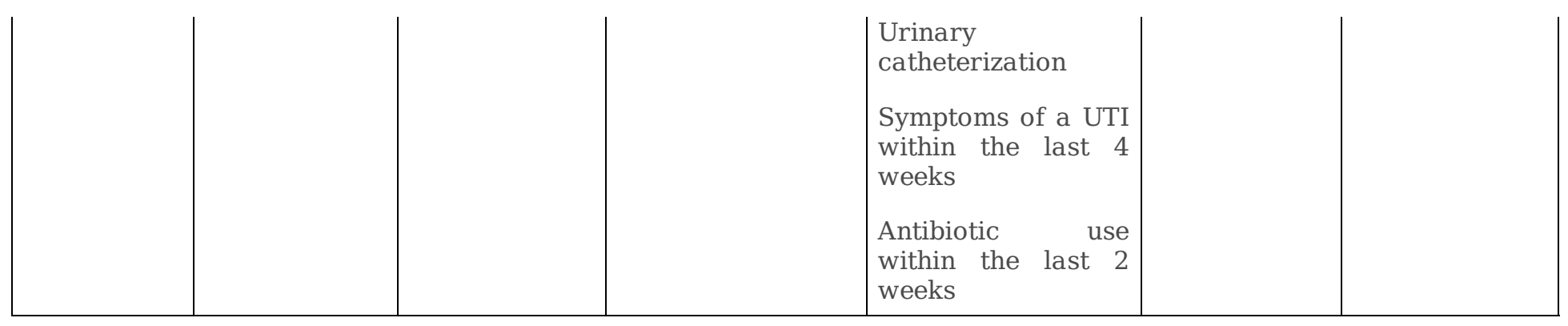

\section{Figures}

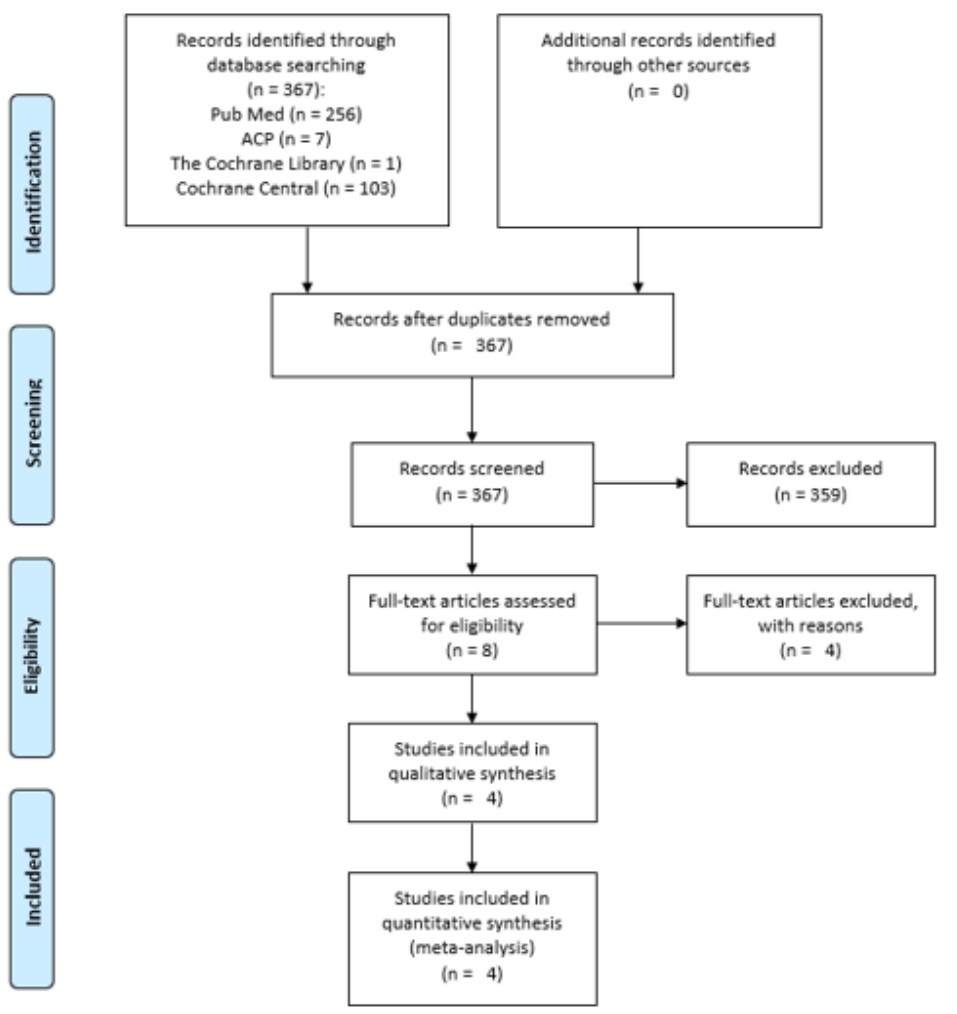

Figure 1

PRISMA Flow Chart of Literature Search 

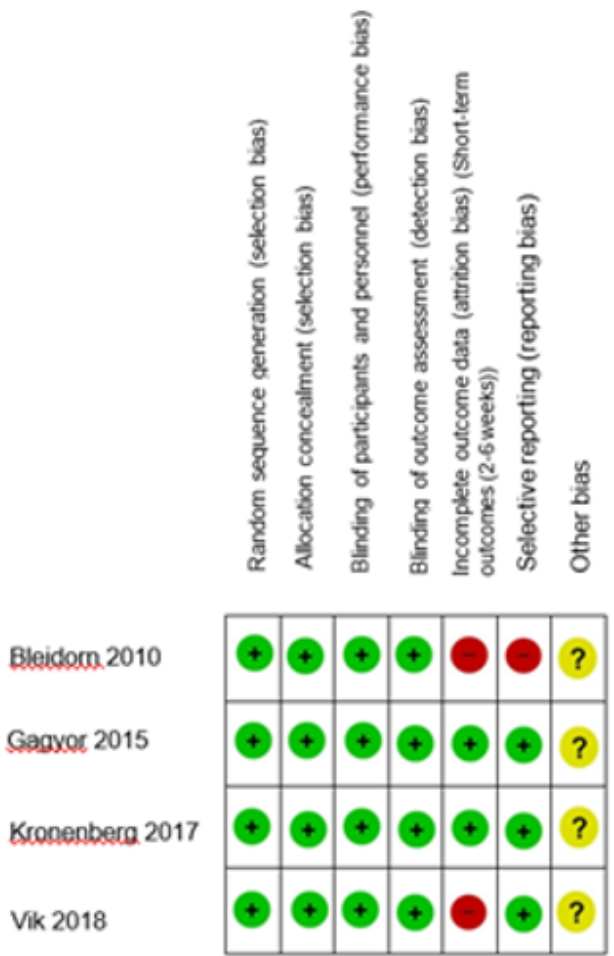

Figure 2

Cochrane risk of bias assessment
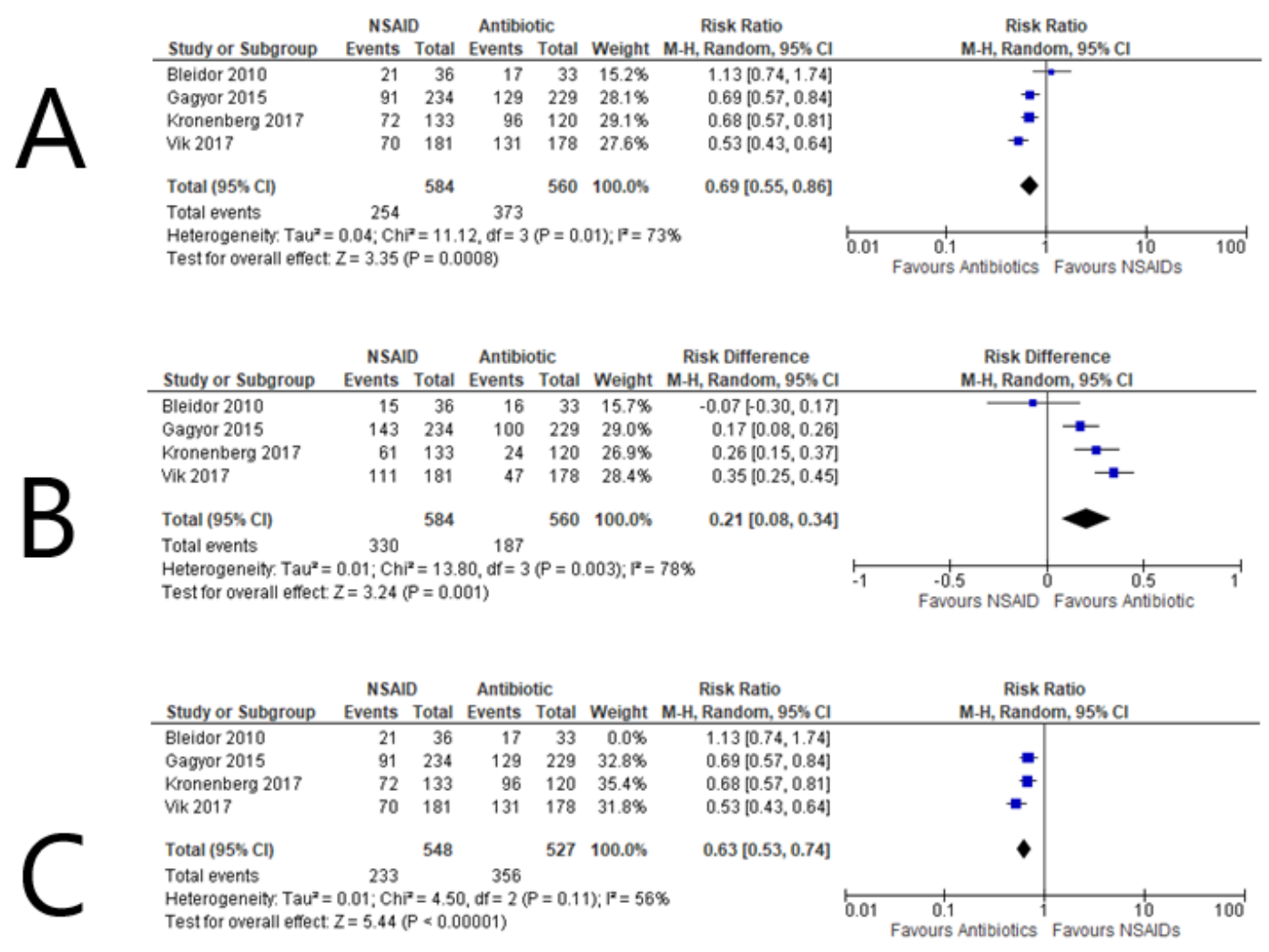

Figure 3

Figure 3A. Forest plot of comparison: Summary of symptomatic cure on Day 3 or 4 of treatment between NSAID and Antibiotic use in women with uncomplicated UTI Figure 3B. Forest plot of comparison: Risk difference between NSAID and Antibiotic use on persistence of symptoms 
on Day 3 or 4 of treatment Figure 3C. Forest plot of comparison: Sensitivity analysis on Day 3 or 4 of treatment between NSAID and Antibiotic use in women with uncomplicated UTI

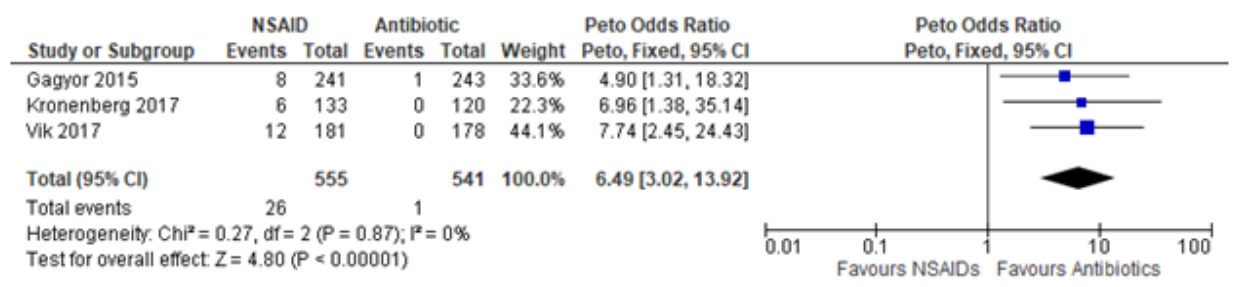

\section{Figure 4}

Forest plot of comparison: Summary of complications [Pyelonephritis and Febrile UTI] in women with uncomplicated UTI randomized to either NSAID or Antibiotic

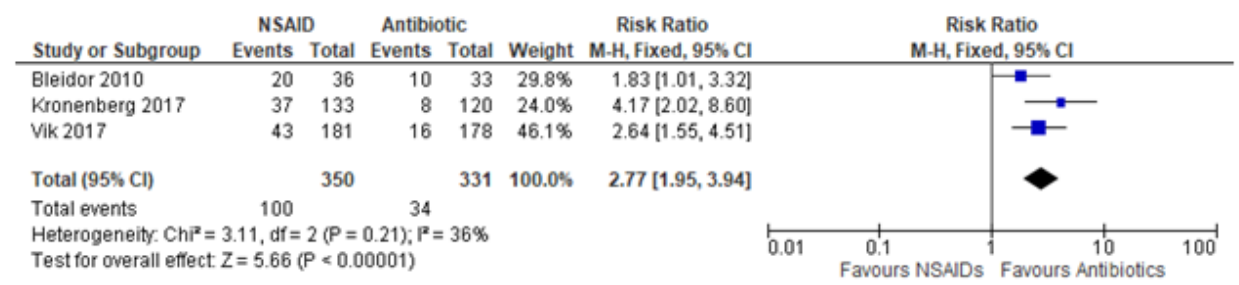

\section{Figure 5}

Forest plot of comparison: Summary of follow-up positive urine cultures [Day 7 to 14] in women with uncomplicated UTI randomized to either NSAID or Antibiotic

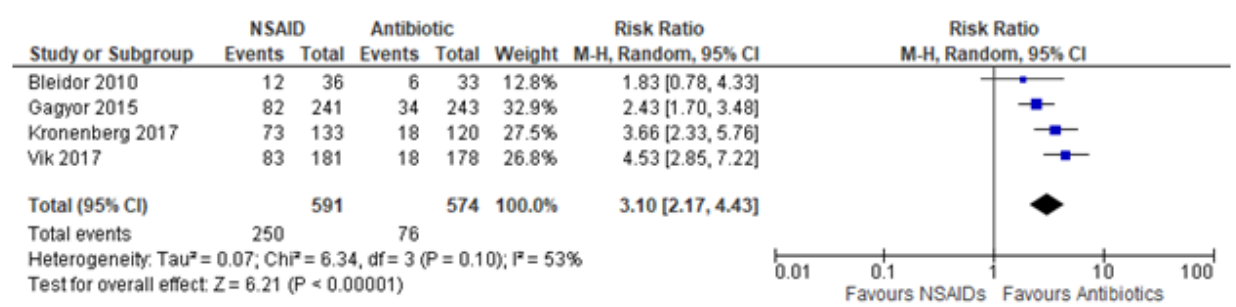

\section{Figure 6}

Forest plot of comparison: Summary of use of secondary antibiotic for persistent or worsening UTI symptoms in women with uncomplicated UTI randomized to either NSAID or Antibiotic

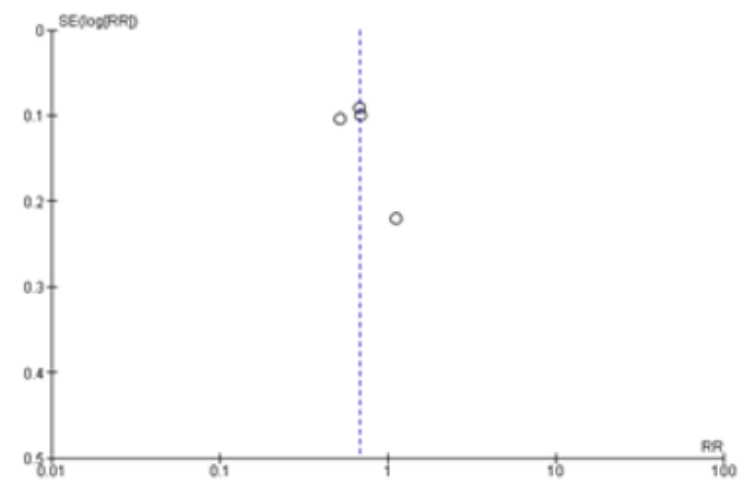


Funnel plot of comparison: NSAID vs antibiotic on Day 3 or 4 of symptomatic cure

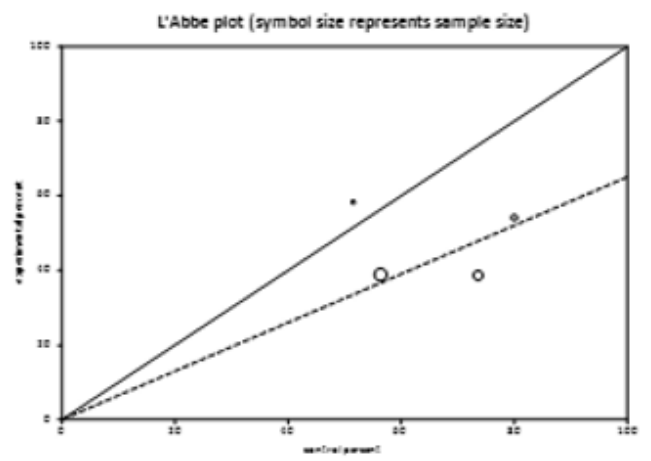

Figure 8

L'Abbe plot: Symptom resolution response rates to treatment vs control 